\title{
PANCREATITIS
}

\section{Pancreatitis: a loss of FGF21 function disease with potential for correction}

A new study shows that deficiency

in pancreatic fibroblast growth

factor 21 (FGF21) is a driving factor in acute and chronic pancreatitis in mice and humans. The new work also demonstrates that FGF21 treatment in various mouse models of pancreatitis alleviated symptoms and prevented onset of the disease, highlighting its clinical potential.

The exocrine pancreas is a source of digestive enzymes that can cause tissue damage and inflammation known as pancreatitis when prematurely activated in the pancreas itself. The exocrine pancreas also expresses FGF21, where it stimulates digestive enzyme secretion by acinar cells. "This project was started based on previous work from our lab and others demonstrating that loss of FGF21 from the pancreas results in an increased susceptibility to acute pancreatitis in mice," says lead author David Mangelsdorf.
The team's previous work also found that FGF21 protection from pancreatitis was due to its ability to mitigate the integrated stress response (ISR) pathway, part of the unfolded protein response (UPR). "This is a key finding because acinar cells synthesize more protein than almost every other cell type in the body, so controlling the UPR is important," explains Mangelsdorf.

In the latest work, using mouse models similar to human disease, including alcohol induced pancreatitis and post-endoscopic retrograde cholangiopancreatography pancreatitis, administration of FGF21 was shown to be effective in treating and preventing pancreatitis. In these mice, as well as samples from patients with acute and chronic pancreatitis, a loss of FGF21 expression was found in the pancreas. "This suggests that pancreatitis is a loss of FGF21 function disease and explains why FGF21 replacement therapy might be effective," reports Mangelsdorf.

Using in vitro experiments, the mechanism of FGF21 loss was attributed to overactivation of the ISR pathway, which induced the expression of ATF3, a transcriptional suppressor of FGF21. Importantly, this mechanism was also confirmed in samples from patients with pancreatitis.

The researchers are now looking towards a clinical trial. "Our findings suggest a new therapeutic use of FGF21, which has already been used in clinical trials for other indications such as obesity," says Mangelsdorf. "Notably, these other indications require chronic use of FGF21, whereas pancreatitis would be a more acute indication with implications for reducing potential adverse events associated with long-term FGF21 use."

Iain Dickson

ORIGINAL ARTICLE Hernandez, G. et al. Pancreatitis is an FGF21-deficient state that is corrected by replacement therapy. Sci. Transl. Med. 525, eaay5186 (2020)

\section{COLORECTAL CANCER}

\section{Anti-tumorigenic endogenous gut bacteria in mice and humans}

The role of the gut microbiota in promoting intestinal tumorigenesis has been well documented, but little is known about the anti-tumorigenic roles of the gut microbiota. In a new study, an endogenous bacterial strain in mice and humans that blocks intestinal tumour growth has been identified for the first time.

"We were puzzled by the observation that germ-free mice can be either protected from or more prone to tumour development depending on the model system," says Maria Rescigno, corresponding author of the new study. By analysing the gut microbiota in a mouse model of colorectal cancer (CRC) and wild-type mice, the researchers identified a strain of Faecalibaculum rodentium (F.PB1) that is underrepresented in the CRC mouse model early in tumorigenesis.

"Epithelial cells undergoing transformation produce different mucins, which create an unfavourable environment for bacteria colonization," says Rescigno. "This situation leads either to the loss of protective strains or the enrichment of tumour-promoting strains." To investigate a protective role for $F$. $P B 1$, the researchers treated the $A p c^{\mathrm{Min} /+}$ mouse model of $\mathrm{CRC}$ and wild-type mice with antibiotics and then F. PB1. Administering F. PB1 4-8 weeks after birth did not affect tumour development, but administration in weeks 8-12 reduced tumour growth in $\mathrm{Apc}^{\mathrm{Min} /+}$ mice, indicating a role for F. PB1 in colorectal tumour growth but not initiation. Next, the researchers showed in $\mathrm{Apc}^{\mathrm{Min} /+}$ mice that the metabolic products of F. PB1, particularly the short-chain fatty acid butyrate, block tumour growth by inhibiting histone deacetylase activity and thereby reducing activation of NFATc 3 and calcineurin, which have a role in cell proliferation. Finally, the researchers used a metagenomic dataset from patients with colorectal adenomas to identify Holdemanella biformis as the human homologue of F. PB1. They showed that the metabolic products of $H$. biformis can reduce tumour cell proliferation, with a corresponding increase in histone acetylation and decrease in NFATc3.

" $F$. PB1 and H. biformis belong to the tumourprotecting bacteria that are lost during intestinal tumorigenesis," concludes Rescigno. Now, the

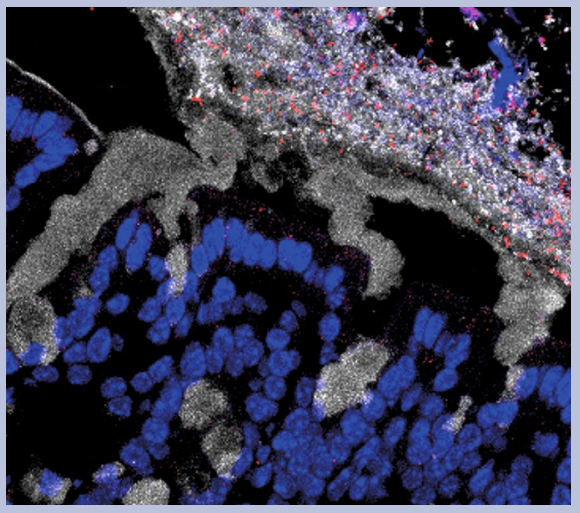

Confocal image showing combined MUC2 immunostaining (grey) and fluorescence in situ hybridization analysis for Faecalibaculum rodentium PB1 (red) in colon tissue of wild-type mice. Nuclei are stained with DAPI (blue). Image courtesy of A. Bertocchi and C. Pozzi, Humanitas University, Italy.

researchers hope to examine whether metabolites other than butyrate are responsible for this anti-tumorigenic effect. "We also want to analyse whether $\mathrm{H}$. biformis can be used as a diagnostic tool or as a probiotic in patients with advanced colorectal adenoma," says Rescigno.

Jordan Hindson

ORIGINAL ARTICLE Zagato, E. et al. Endogenous murine microbiota member Faecalibaculum rodentium and its human homologue protect from intestinal tumour growth. Nat. Microbiol. https://doi.org/10.1038/s41564-019-0649-5 (2020) 